\title{
Immunology and Immunotherapy
}

Open Access

\section{Evaluation of Cross-Reactivity of Suidasia pontifica with Allergens Blo t 5 from the Blomia tropicalis Mite}

\section{Francisca das Chagas Sobral Silva ${ }^{1,2^{*}}$ (D), Daniel Vasconcelos $R$ Silva $^{2}$ (D), Anderson Bruno Matos ${ }^{2}$ (D), Andrea Medeiros Salgado ${ }^{\text {(D) }}$ and Maria Queiroz ${ }^{2}$ (D)}

${ }^{1}$ Department of Biochemical Engineering, School of Chemistry, Technology Center, Universidade Federal do Rio de Janeiro, Rio de Janeiro, Brazil

${ }^{2}$ Laboratory of Extracts Allergens $M Q$, Research and Development, Rio de Janeiro, Brazil

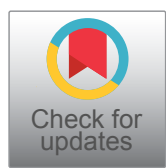

*Corresponding author: Francisca das Chagas Sobral Silva, Department of Biochemical Engineering, School of Chemistry, Technology Center, Universidade Federal do Rio de Janeiro; Laboratory of Extracts Allergens, Rio de Janeiro, Brazil, Tel: $+5521981204546$

\begin{abstract}
Background: The immunological cross-reactivity between different species of dust mites such as Suidasia pontifica and Blomia tropicalis is a factor that can favor an immunotherapeutic treatment, having so great clinical relevancy. However, the pretreatment of the raw extract can potentialize its response as in the cross-reactivity analysis. Therefore, this paper seeks to pre-treat the Suidasia pontifica extract in order to obtain protein fractions by the "salt out" method and then evaluate the presence of cross-reactivity for the Blo 5 allergen of Blomia tropicalis.
\end{abstract}

Method: Suidasia pontifica extracts were obtained by the protein precipitation with ammonium sulfate in percentages $0-20 \%, 20-40 \%, 40-60 \%, 60-80 \%, 80-100 \%(w / v)$ at $0{ }^{\circ} \mathrm{C}$. Precipitated fractions were evaluated by protein content, characterized by electrophoresis, and applied in Western Blot and ELISA tests, with positives and negatives controls using the recombinant antigen Blo 5 .

Results: With salt saturation level of $80 \%(w / v)$, it was achievable to efficiently separate proteins in the raw extract of Suidasia pontifica, resulting in a total content of $13.61 \mathrm{mg}$ $\mathrm{mL}^{-1}$. The immunoassay ELISA exposed the existence of cross-reactivity for Blo 5 allergen, for crude extract, with allergenic potency of $2.797 \mathrm{ng} \mathrm{mL}^{-1}$. This result was also confirmed by stains obtained in the blot in about 15-16 kDa. For fractions obtained between the percentages of 20 to $100 \%$ $(\mathrm{w} / \mathrm{v})$ of salt saturation and power was below $1.93 \mathrm{ng} \mathrm{mL}^{-1}$, at the border of the established limit of detection.

Conclusion: The "salt out" method is efficient at obtaining concentrated fractions of proteins, as observed when applied in Suidasia pontifica, extract, this being susceptible to cross-interactions, suggesting the possibility of this mite to act as an adjunct in immunotherapy on formulations for desensitization of allergic individuals.

\section{Introduction}

Dust mites are responsible for pathologies in the respiratory tract triggering cases of allergies in atopic individuals $[1,2]$. The dust mite species Suidasia pontifica, (S. pontifica = SP) can be found in domestic dust, in addition to grains and stored spices [3]. This mite is specially responsible for triggering allergic reactions mediated by specifics immunoglobulin [4]. Scientific studies show the cross-reactivity between dust mites such as Dermatophagoides pteronyssinus, Dermatofagoides farinae, Tyrophagus putrescentiae, Blomia tropicalis, Suidasia medanensis, and others species [5-7]. Studies that evaluate the cross-reactivity usually initiate with the preparation of the raw extract of the mite of interest $[8,9]$ however, there is an absence of information that relates the efficiency in the preparation stage of these extracts with the performance of the cross-reactivity power. A novel investigation here is proposed, considering the relation between proteic concentration, obtained by fractional precipitation, and cross-reactivity for Blo t 5 allergen (B. tropicalis). The relevance of the cross-reactivity study between mite species $S$. pontifica and $B$. tropicalis can fulfill studies gaps that don't ap-

Citation: Silva FCS, Silva DVR, Matos AB, Salgado AM, Queiroz M (2020) Evaluation of Cross-Reactivity of Suidasia pontifica with Allergens Blo 5 from the Blomia tropicalis Mite. Int J Immunol Immunother 7:056. doi.org/10.23937/2378-3672/1410056

Accepted: November 28, 2020; Published: November 30, 2020

Copyright: (c) 2020 Silva FCS, et al. This is an open-access article distributed under the terms of the Creative Commons Attribution License, which permits unrestricted use, distribution, and reproduction in any medium, provided the original author and source are credited. 
proach specifically the Blo t 5 allergen for this Suidasia species, which can also be interesting to evaluate this activity by optimization of "salting out" method. That is possible because the precipitation method, using ammonium sulfate, allows to separate proteins of different sizes, and consequently allergens with distinct molecular masses. Thus, it is expected amplification of cross-reactivity signal due to the optimized allergenic protein concentration of the extract under analysis.

\section{Materials and Methods}

\section{Suidasia pontifica mite protein extraction}

$1.0 \mathrm{~g}$ of the Suidasia pontifica mite mass was macerated in the presence of $60.00 \mathrm{~mL}$ of sodium chloride extracting saline solution in a $\mathrm{pH} 9$ alkaline medium. After $18 \mathrm{~h}$ of stirring under refrigeration at $8{ }^{\circ} \mathrm{C}$, the extract was centrifuged and the supernatant filtered in $0.45 \mu \mathrm{m}$ pre-filter. The protein content was determined by the Lowry method [10] and total carbohydrate as in [11].

\section{Salting out precipitation}

$50.00 \mathrm{~mL}$ of the crude extract of the Suidasia pontifica mite was subjected to precipitation by sequential fractionation with ammonium sulfate in the salt saturation percentages of $0-20 \%(2.82 \mathrm{~g}), 20-40 \%(3.02 \mathrm{~g})$, $40-60 \%$ (3.25g), 60-80\% (3.50g) and $80-100 \%$ (3.80g) according to the procedure indicated by the literature [12]. The system was kept in an ice bath under agitation for $40 \mathrm{~min}$. With the gradual increase in the salt mass, the solubilization time doubled. Afterwards, the extract was centrifuged at $5724 \mathrm{xg}$ under $2{ }^{\circ} \mathrm{C}$ refrigeration for 30 minutes.

The pellets obtained after centrifugation were resuspended in $5.00 \mathrm{~mL}$ purified water and the aqueous supernatant returned to the ice system, for the other fractionation sequences. The precipitated fractions obtained for each percentage were stored at $-20^{\circ} \mathrm{C}$, for further characterization.

\section{Protein content determination}

The precipitate protein contents for each fraction of saturation with the salt, $\left(\mathrm{NH}_{4}\right)_{2} \mathrm{SO}_{4}$, were analyzed according to the Lowry method. For this, bovine serum albumin (Sigma-Aldrich) was used as a standard protein and an analytical curve was built for a working range between $5.0 \mu \mathrm{g} \mathrm{mL}^{-1}$ to $100.0 \mu \mathrm{g} \mathrm{mL}$. The absorbance readings were performed at $750 \mathrm{~nm}$ using a UV-Visible spectrophotometer (Spectrophotometer SP 1102 - Bel Photonics - Brazil).

\section{Immunoblotting}

The proteins of the extracts were separated by Electrophoresis SDS-PAGE, in gradient gel $5-12.5 \%$, and transferred to $0.45 \mu \mathrm{m}$ pore nitrocellulose membrane (Bio-Rad). The membrane and gel containing the allergens were arranged in sandwich form, covered with a paper filter and compressed with polyurethane sponges, on the transfer support. All material was previously soaked in saline buffer solution at $\mathrm{pH} 7.2$, consisting of $25 \mathrm{mM}$ of Tris-Base, $192 \mathrm{mM}$ of Glycine and $20 \%(\mathrm{v} / \mathrm{v})$ of methanol. The slow transfer conditions were applied with constant amperage of $18 \mathrm{~V}, 10 \mathrm{~mA}$ and $70 \mathrm{~W}$, for 18 $\mathrm{h}$ to $8{ }^{\circ} \mathrm{C}$. To confirm the transfer of proteins from gel to nitrocellulose membrane, ponceau-S $0.2 \%$ dye solution $(\mathrm{m} / \mathrm{v})$ was added.

Serum from 15 individuals allergic to Blomia tropicalis mites confirmed after evaluation by immediate reading skin test (Prick Test) was used for the antigen-antibody reactivity test. The membrane containing the proteins was initially blocked, with bovine serum albumin solution $(0.5 \%)$ in saline buffer (TBS) for $1 \mathrm{~h}$, under gentle agitation. Prior blockade was necessary in order to avoid nonspecific connections and background spots. After this period, the membrane was washed with TBS-T buffer containing $0.1 \%$ Tween and incubated in $20.0 \mathrm{~mL}$ of the serum pool containing IgE (1:10) for $5 \mathrm{~h}$, under mild agitation. At the end of the established period the membrane was washed again with Tween solution for removal of excess primary antibody not attached to the membrane. The secondary antibody, human anti-IgE, specific epsilon chain, conjugated to the enzyme peroxidase (Sigma-Aldrich), was diluted 1:200 in TBS buffer. The membrane was incubated in this solution for a period of $4 \mathrm{~h}$ under slight agitation. The presence of effective antibody antigen bonds was investigated using the Amplifled Kit Opti-4CN /Bio-Rad).

\section{Enzyme-linked immunosorbent assay (ELISA) characterization}

The extract of the mite Suidasia pontifica was analyzed by the ELISA method - Immunosorption Assay attached to the enzyme, by the construction of a standard curve of the allergen Blo $t 5$ (ELISA kit; recombinant Blo t 5 prepared in $1 \%$ BSA $/ 50 \%$ glycerol/PBS, $0.22 \mu \mathrm{m}$ filtered, preservative free, $\mathrm{pH} 7.4$, Indoor Biotechnologies-USA), according to the literature Luczynska, et al. [13]. The allergenic protein concentration was built using the four-parameter logistic curve (4PL). Briefly, 100 $\mu \mathrm{L}$ of anti Blo t 5, (Monoclonal antibody, Indoor Biotechnologies- USA), was added to the polystyrene plate microplates (USA) as a capture molecule. After 18 hours of incubation at $8{ }^{\circ} \mathrm{C}$ the plate was washed with PBS-T saline buffer, the excess buffer was removed and blocked with $1 \%(w / v)$ BSA solution. After 1 hour of blocking, the plate was washed and the standard Blo 5 antigen ( $\mathrm{rBlo}$ t 5 Standard- Indoor Biotechnologies- USA) was added.

The standard curve was built in a working range between $0.098 \mathrm{ng} \mathrm{mL}^{-1}$ to $120 \mathrm{ng} \mathrm{mL}^{-1}$ for the allergen Blo $\mathrm{t} 5$, evaluating the cross-reactivity with proteins present in the mite SP extract. After $18 \mathrm{~h}$ of incubation with the standard antigen the plates were washed with $0.1 \mathrm{~mol}$ $\mathrm{L}^{-1}$ phosphate saline buffer. For the subsequent step, 
$100 \mu \mathrm{L}$ of solution containing Biotinylated monoclonal antibody, anti Blo t 5 (Indoor Biotechnologies- USA), was added. After $1 \mathrm{~h}$ of incubation the plate was washed again and $100 \mu \mathrm{L}$ of ABST substrate (2,2-Azino-bis (3-ethylbenzothiazoline-6-sulfonic. Finally, the substrate reaction was stopped with $1 \%$ SDS solution $(w / v)$. The readings were performed at $450 \mathrm{~nm}$ using a plate reader model dr-200bn from DIATEK.

\section{Results and Discussion}

The electrophoretic run shown in Figure 1 shows the constituent proteins profile for the crude extract of the Suidasia pontifica mite, with bands marked at approximately 14-16 kDa, 24-25 kDa and 60-70 kDa.

The protein content obtained for the crude extract was $1.45 \mathrm{mg} \mathrm{mL}^{-1}$. The levels for the different percentages of proteins are shown in Figure 2A. One can observe that the protein extract fractionated with $80 \%(\mathrm{w} / \mathrm{v})$ of ammonium sulfate, showed better separation of low molecular weight proteins, $15 \mathrm{kDa}$ (Figure 2B) with a maximum content of $13.62 \mathrm{mg} \mathrm{mL}^{-1}$ (Figure $2 \mathrm{~A}$ ). At the beginning of the fractionation, Figure $2 B$, in the percent-

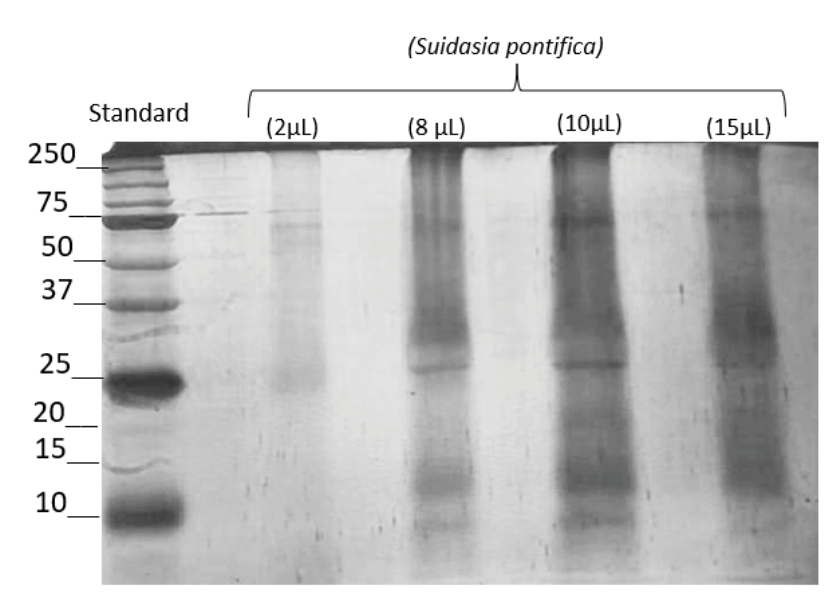

Figure 1: Electrophoresis gel $12 \%$ Acrylamide/Bisacrylamide in denaturing conditions, for crude extract of the Suidasia pontifica mite for protein load between $2 \mu \mathrm{L}$ and $15 \mu \mathrm{L}$. age of $20-40 \%(\mathrm{w} / \mathrm{v})$ there was a separation of proteins from larger ones (above $40 \mathrm{kDa}$ ), due to the smaller area of water solvation. As the ionic force increases, the tendency of smaller protein precipitation decreases, which can be observed in the electrophoresis gel (Figure $2 B)$ in the fractions of $80(w / v)$ and $100 \%(w / v)$. This can be justified, by the simple fact that smaller proteins have greater surfaces of solvation by the water, being necessary the increase of the concentration of the ions coming from the salt to capture water molecules that surround the proteins.

The immunoblotting for the protein fractions separated by electrophoresis, Figure 3, showed positive reactivity for allergenic proteins in the region of molecular mass between 14-16 kDa. The spots obtained in the blot are a strong indication that in this region reactivity may be occurring between antigen (SP extract allergen protein) and antibody present in the serum pool. From this result, the reactivity was evaluated by ELISA.

These results were confirmed, considering that crude extract, without fractionation, analyzed by the

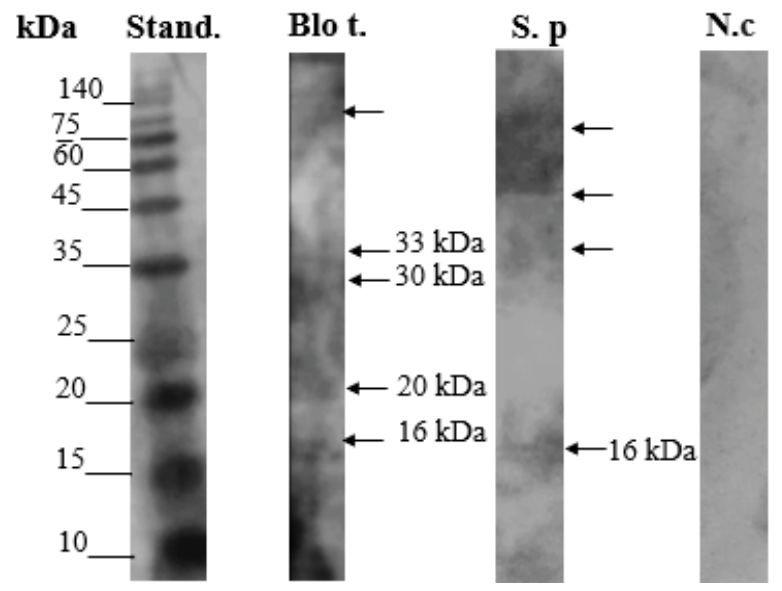

Figure 3: Immunoblotting for serum pool of individuals allergic to Blomia tropicalis. Blo t: Blomia tropicalis positive control; S.p: Suidasia pontifica; N.c: Negative control Bovine serum albumin - BSA.
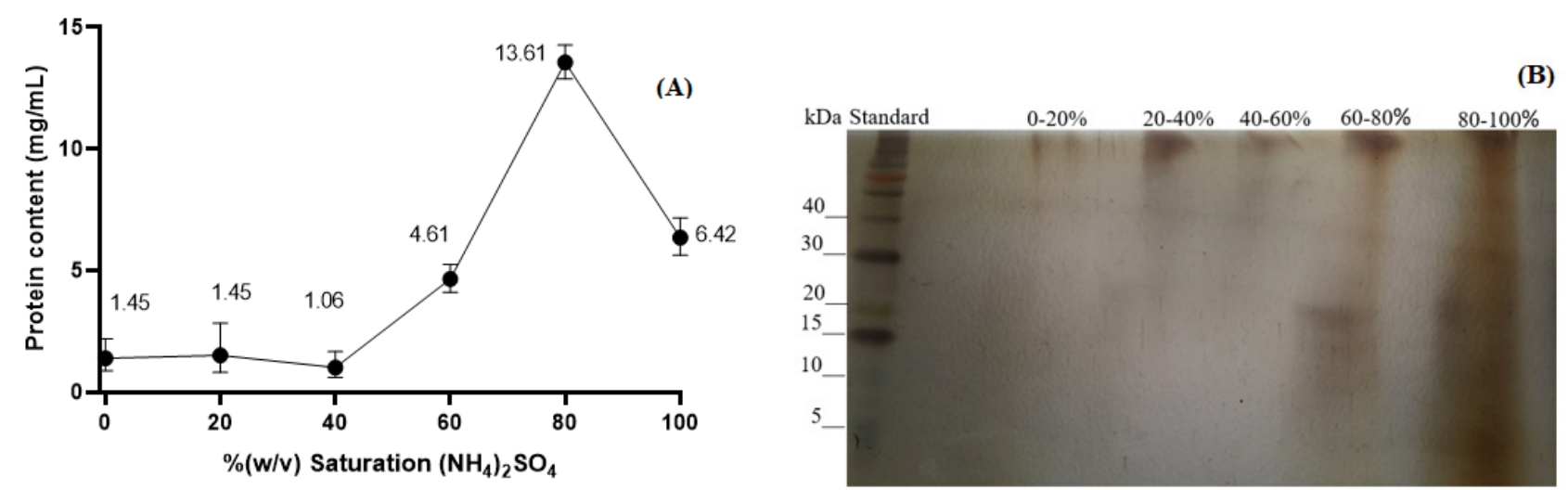

Figure 2: A) Protein content in $\mathrm{mg} \mathrm{mL}^{-1}$ of the Suidasia pontifica mite extract obtained for each fractionation sequence by the method "salting out" at $\sim 0{ }^{\circ} \mathrm{C}$; B) Electrophoresis gel in denaturing conditions for the fractions collected from the SP extract during the fractionation process. 
ELISA method, presented an allergenic potency of 2.797 $\mathrm{ngmL}^{-1}$, which indicates cross-reactivity between Suidasia pontifica with the allergen Blo t 5 (14-15 kDa) of the Blomia tropicalis mite.

Further studies on the medium activation of cells (mast cells, basophils, etc.) to release histamine, should be investigated for better clarification in order to assess the allergenic potential of the mite antigens SP.

For all other obtained fractions in the different percentages of ammonium salt between 20 to $100 \%(\mathrm{w} / \mathrm{v})$ ) the allergenic potency with cross-reactivity to Blo t 5 showed concentrations below $1.93 \mathrm{ng} \mathrm{mL}^{-1}$.

In general, the results obtained show the efficiency of this separation technique by the fractionation method by "salting out", however, there is a limitation when low concentrations of the allergen ( 14-16 kDa). The results indicate that the extract of the Suidasia pontifica mite, has a potential for desensitization in immunotherapy procedures, for atopic individuals with predisposition to allergies such as asthma, rhinitis. These results are in agreement with [9], which identified proteins present in the extract of the Suidasia medanensis mite when in contact with serum IgE from individuals allergic to it. Molecular weights ranged from 7.5 to $105 \mathrm{kDa}$. The authors report antigen reactivity to the protein bands at 30-31 kDa, 24.5 kDa, 21.47 kDa and $58 \mathrm{kDa}$, with other allergens $(7.5 ; 14.5 ; 16 ; 27.5 ; 35.5 ; 41-42 ; 66 ; 75$ and $105 \mathrm{kDa}$ ) were detected at lower frequencies and that by immunoblot inhibition it was possible to observe the ability of Blomia tropicalis extract to inhibit IgE binding of the serum pool to Suidasia medanensis.

\section{Conclusion}

The application of the "salting out" method is a viable alternative for the concentration of proteins from dust mite extracts. The starting crude extract can be optimized as to its extraction method so that the allergenic fractions, reactive to Blo $t 5$, can be obtained in the different percentages of saturation. It is also considered that the Suidasia pontifica mite presents specific cross-reactivity to the allergen Blo $t 5$, of the mite Blomia tropicalis. This suggests that "in vivo" immunotherapy procedures for Blomia tropicalis may be followed by decreased immunological reactivity for Suidasia pontifica in patients allergic to dust mites. However, these results will be much more clear when animal testing is performed.

\section{Acknowledgement}

Thanks to the report of allergenic extracts for the financial support.

We thank the biological sensor laboratory for technical support.

\section{Conflict of Interests}

The authors have not declared any conflict of inter- ests.

\section{References}

1. Mondal Prit, Debarati Dey, Nimai Chandra Saha, Saibal Moitra, Saibal Moitra, et al. (2019) Investigation of house dust mite induced allergy using logistic regression in West Bengal, India. World Allergy Organization Journal 12: 1-9.

2. Yuriev S, A Kurchenko, S Zubchenko, V Tsaryk, L DuBuske, et al. (2020) Diagnostic feature of house dust mite sensitization. Ann Allergy Asthma Immunol 125: S17-S52.

3. Thomas Wayne R, Belinda J Hales, Wendy Anne Smith (2010) House dust mite allergens in asthma and allergy. Trends in Molecular Medicine 16: 321-328.

4. Ngu Mark Angelo V, Bergantin Jose H, Ramos JDA (2019) Development of a gold nanoparticle-labeled sandwich format lateral flow immunoassay kit for the detection of tropical house dust mite Suidasia pontifica. Protein Pept Lett 26: 357-363.

5. Yu Ching Hsiang, Jaw Ji Tsai, Yi Hsueh Lin, Sheng Jie Yu, En Chih Liao (2020) Identification the cross-reactive or species-specific allergens of Tyrophagus putrescentiae and development molecular diagnostic kits for allergic diseases. Diagnostics.

6. L Liu, Xiaoqing, Peiyan Zheng, Song Guo Zheng, Yingying Zhai, et al. (2019) Co-sensitization and cross-reactivity of Blomia tropicalis with two Dermatophagoides species in guangzhou, China. Journal of Clinical Laboratory Analysis.

7. Lahiani Sadjia, Marie Eve Dumez, Souad Khemili, Idir Bitam, Dimitri Gilis, et al. (2019) Cross-reactivity between major IgE epitopes of family 5 allergens from Dermatophagoides pteronyssinus and Blomia tropicalis. International Archives of Allergy and Immunology 178: 10-18.

8. Mihos, Francisca das Chagas Sobral Silva, Patricia Ribeiro Pereira, Anderson Bruno de Almeida Matos, Caio Velloso Mergh, et al. (2017) Study of protein profile and immunochemical reactivity for extracts of $D$. Farinae, $D$. Pteronyssinus and $\mathrm{B}$. Tropicalis mites in the city of rio de janeiro, Brazil. Arquivos de Asma, Alergia e Imunologia 1: 379-386.

9. Puerta L, Lagares A, Mercado D, Fernandez-Caldas E, Caraballo L (2005) Allergenic composition of the mite Suidasia medanensis and cross-reactivity with Blomia tropicalis. Allergy 60: 41-47.

10. Lowry O, Rosebrough J, Lewis A, Randal R (1951) Medicion de proteinas con el reactivo de fenol Folin. J Biol Chem 193: $265-275$.

11. Gail Lorenz Miler (1959) Use of dinitrosalicylic acid reagent for determination of reducing sugar use. Anal Chem 31: 426-428.

12. Scopes RK (1994) Protein purification: Principles and practice. Springer.

13. Luczynska CM, Arruda LK, Platts-mills TAE, Lopez M, Chapman MD, et al. (1989) A two-site monoclonal antibody ELISA for the quantification of the major Dermatophagoides spp . allergens, Der $p$ I and Der f I. Journal of Immunological Methods 118: 227-235. 